\title{
Effectiveness of early postoperative oral hydration after colorectal surgery with general anesthesia: A prospective randomized controlled trial
}

\section{Xiaorong Yin}

Sichuan University West China Hospital

Lei Yang

Sichuan University West China Hospital

Shunju Xiang

Sichuan University West China Hospital

Yixuan Wu

Sichuan University West China Hospital

Qian Li ( $\nabla$ sculiqian@foxmail.com )

Sichuan University West China Hospital https://orcid.org/0000-0003-3301-4284

Research article

Keywords: Early oral hydration, colorectal surgery, general anesthesia, randomized controlled trial

Posted Date: November 26th, 2019

DOI: https://doi.org/10.21203/rs.2.17432/v1

License: (c) (1) This work is licensed under a Creative Commons Attribution 4.0 International License. Read Full License 


\section{Abstract}

BACKGROUND: Over the last decades, studies have already demonstrated that early feeding in patients after elective colorectal surgery yielded a shorter length of hospital stay and did not cause additional risk for adverse events. However, the optimal timing for beginning oral hydration after colorectal surgery under general anesthesia remains controversial. Therefore, we conducted the study to evaluate the effects of early oral hydration $(\mathrm{EOH})$ versus traditional oral hydration $(\mathrm{TOH})$ on thirst and clinical safety outcomes after colorectal surgery under general anesthesia.

METHODS: This prospective randomized controlled trial of 1,000 patients with American Society of Anesthesiologists I- III who underwent colorectal surgery under general anesthesia were randomly assigned to the $\mathrm{EOH}$ group (given $0.5 \mathrm{ml} / \mathrm{kg}$ water after recovery from general anesthesia) or TOH group (fasting and water deprivation until postoperative intestine function recovery). The primary outcome was thirst scale, and secondary outcomes were discomfort score, nausea and vomiting score, and safety outcomes.

RESULTS: Of the 1,000 patients who underwent randomization at initial stage, 27 were excluded in $\mathrm{EOH}$ group because of refusal $(n=22)$ or nausea and vomiting before hydration $(n=5)$. Demographic and operative data were similar, but not statistically significant $(P>0.05)$. Patients who received EOH were associated with lower thirst score than with $\mathrm{TOH}(\mathrm{EOH} 45.70 \pm 24.51$ vs. TOH $62.20 \pm 23.99 ; \mathrm{P}<0.001)$ and oropharyngeal discomfort scale $(\mathrm{EOH} 3.71 \pm 8.49$ vs. $\mathrm{TOH} 6.18 \pm 11.89, \mathrm{P}<0.0001) 30$ min after drinking. No significant differences were found for the time of intestinal movements $(\mathrm{EOH} 73.37 \pm 34.49 \mathrm{~h}, \mathrm{TOH}$ $70.56 \pm 31.71, \mathrm{P}=0.187$ ) and for $\mathrm{EOH}$ and $\mathrm{TOH}$ for the risk of nausea and vomiting at the postoperative period $(P>0.05)$ and other complications $(P>0.05)$.

CONCLUSIONS: The findings suggested the safety outcomes for mild EOH on patients after colorectal surgery under general anesthesia. Patients who received EOH could have significantly reduced degree of thirst scale and oropharyngeal discomfort scale 30 min after drinking. Trial registration: CHiCTR, CHiCTRTRC-13003097. Registered 11 March2013, http://www.chictr.org.cn/showproj.aspx?proj=6462.

\section{Background}

Colorectal cancer is one of the most common gastrointestinal tumors, and surgery is the preferred treatment [1]. Optimizing perioperative care can decrease postoperative complications, accelerate the recovery of gastrointestinal function, and improve patients' comfort and satisfaction [2]. Traditional postoperative management of colorectal surgeries has been to keep the patient "nil by mouth" and provide gastric decompression via nasogastric tube to prevent postoperative complications, such as ileus, anastomotic fistula, intra-abdominal infection, nausea, and vomiting [3,4]. This oral hydration practice is still widely used for patients who underwent colorectal surgery under general anesthesia [5], with which clear liquids should be given on the postoperative day following the return of bowel 
movements and the diet should be gradually accelerated to a regular diet according to patients' tolerance after passage of flatus [6].

However, over the last decades, studies have already demonstrated that early feeding in patients after elective colorectal surgery yielded a shorter length of hospital stay and did not cause additional risk for adverse events, including anastomotic dehiscence, pneumonia, wound infection, rate of nasogastric tube reinsertion, vomiting, or mortality [4,7-12]. Our previous study suggested that starting oral hydration after recovery from general anesthesia was safe, with decreased thirst scale and oropharyngeal discomfort scales, and higher satisfaction for patients undergoing non-gastrointestinal surgery [13]. However, the optimal timing for beginning oral hydration after colorectal surgery under general anesthesia remains controversial.

Therefore, the current randomized controlled trial was conducted to determine whether early oral hydration $(\mathrm{EOH})$ after recovery from general anesthesia in the postanesthesia care unit (PACU) is superior to traditional oral hydration $(\mathrm{TOH})$ for patients who underwent colorectal surgery.

\section{Methods}

This prospective randomized controlled trial was approved by the Ethics Committee of Clinical Trials and Biomedical, West China Hospital, Sichuan University (Date: 2012, Number: 220). This study was registered in Chinese Clinical Trial Registry at Match 2013 (Registration number was CHiCTR-TRC13003097). Written informed consent was obtained from all patients.

\section{Trial Design and Randomization}

This current randomized, controlled single-center trial compared EOH with $\mathrm{TOH}$ in patients after colorectal surgery under general anesthesia. The intervention process was open labeled, while the outcome evaluation process was blinded. Patients who met the inclusion criteria were randomly assigned into the $\mathrm{EOH}$ or TOH group with 1:1 ratio using a computer-generated random number using SPSS 18.0 (SPSS Inc., Chicago, IL).

\section{Participants}

One thousand patients with American Society of Anesthesiologists (ASA) I-III grades who underwent colorectal surgery under general anesthesia in the PACU between March 2013 and March 2014 in the West China Hospital, Sichuan, China, were recruited. The inclusion criteria were patients undergoing selective colorectal surgery; ASA score I-III; patients with normal mental status, cough and swallowing reflex; myodynamia level $\mathrm{V}$ in four limbs; and last analgesic drugs were given more than $20 \mathrm{~min}$. The exclusion criteria were patients with intestinal obstruction, intestinal fistula, difficulty swallowing, diabetes, mellitus, or nausea and vomiting. 


\section{Intervention and Outcomes Measures}

Electrocardiogram, pulse oximetry readings, and noninvasive blood pressure were routinely monitored after patients were sent to the PACU. The patients in the EOH group were instructed to drink water 0.5 $\mathrm{ml} / \mathrm{kg}$. The $\mathrm{TOH}$ group received oral water until the postoperative ileus resolves (passage of flatus). The primary outcome was thirst scale, and secondary outcomes were discomfort score, nausea and vomiting score, and safety outcomes. The outcomes of thirst and discomfort scores, nausea and vomiting scores, number of patients expressing a desire to drink water were recorded in the PACU immediately after recovery from general anesthesia and $30 \mathrm{~min}$ later (30 min after the patients in the $\mathrm{EOH}$ group drink water or the comparable time point for the patients in the $\mathrm{TOH}$ group). The thirst score and oropharyngeal discomfort scale were accessed using a verbal numeric scale $(0=$ no thirst/uncomfortable/unsatisfied, $100=$ most thirst/most comfortable/very satisfied). Further, the time of intestinal movements and incidences of in-hospital stoma complications in the $\mathrm{EOH}$ and $\mathrm{TOH}$ groups were recorded.

\section{Sample size and statistical Analysis}

We assumed that type I error $\alpha$ and type II error $\beta$ were 0.05 and 0.2 , respectively. According to the pilot study, we set the $\mu \mathrm{t}, \mu \mathrm{c}$ and margin as $0.3,0.7$ and 0.2 . A total of 684 participants at least were calculated to be included in the study. The data collected are presented as mean \pm standard deviation or events (percentage) for continuous and categories data, respectively. The comparison results between $\mathrm{EOH}$ and $\mathrm{TOH}$ were calculated using $\chi 2$ or $t$ tests. The statistical analysis plan specified two-sided analysis, and a $P$ value less than 0.05 was considered to indicate significant differences between $\mathrm{EOH}$ and TOH. All statistical analyses were conducted using SPSS 18.0 (SPSS Inc., Chicago, IL).

\section{Results}

The flow chart of the study is presented in Fig. 1. A total of 1,000 patients who had undergone colorectal surgery under general anesthesia from March 2013 and March 2014 underwent randomization (500 to the $\mathrm{EOH}$ group and 500 to the $\mathrm{TOH}$ group) at one center in West China Hospital, Sichuan, China. Table 1 summarizes the baseline demographic and clinical characteristics of the two groups. A total of 27 patients were lost to follow-up in the EOH group because of refusal to drink $(n=22)$ or experienced nausea and vomiting $(n=5)$ before drinking. Finally, 973 patients were available for this study, and no significant differences between EOH and TOH were found for the mean age $(P=0.091)$, sex $(n=0.059)$, weight $(P=0.784)$, anesthesia type $(P=0.063)$, ASA scale $(P=0.178)$, surgical type $(P=0.302)$, intraoperative blood loss $(P=0.784)$, and intraoperative fluid infusion $(P=0.860)$. Further, the intraoperative drug use between groups was not statistically significant for most drugs, while the use of dexamethasone in the $\mathrm{EOH}$ group was significantly high than in the $\mathrm{TOH}$ group (Table 2).

Table 3 shows the results of thirst score, oropharyngeal discomfort scale, and time of intestinal movements. No significant differences between the two group were noted in the thirst score $(P=0.094)$ 
and oropharyngeal discomfort score $(P=0.224)$ before drinking. Furthermore, patients in the EOH group had significantly reduced thirst score than those in the TOH group (EOH $45.70 \pm 24.51$ vs. TOH $62.20 \pm 23.99, \mathrm{P}<0.001)$ after $30 \mathrm{~min}$ of oral hydration. Moreover, oropharyngeal discomfort score of the patients in the $\mathrm{EOH}$ group was significantly lower than those in the $\mathrm{TOH}$ group after $30 \mathrm{~min}(\mathrm{EOH}$ $3.71 \pm 8.49$ vs. $\mathrm{TOH} 6.18 \pm 11.89, \mathrm{P}<0.0001)$. Finally, no significant difference was found between groups in terms of time of intestinal movements $(\mathrm{EOH} 73.37 \pm 34.49 \mathrm{~h}, \mathrm{TOH} 70.56 \pm 31.71, \mathrm{P}=0.187)$.

Table 4 summarizes the incidence of nausea and vomiting after drinking at different time points. The risk of nausea (EOH: $1.9 \%$ vs. TOH: $2.8 \% ; \mathrm{P}=0.403$ ) and vomiting (EOH: $0.4 \%$ vs. TOH: $0.0 ; \mathrm{P}=0.234$ ) after 30 min of drinking between $\mathrm{EOH}$ and $\mathrm{TOH}$ groups were not statistically significant. Furthermore, no significant differences were noted between groups for the rate of nausea $(\mathrm{EOH}: 1.1 \%$ vs. $\mathrm{TOH}: 2.0 \% ; \mathrm{P}=$ 0.301 ) and vomiting (EOH: 0.0 vs. TOH: 0.0 ) after leaving the PACU. Moreover, no significant differences were noted between groups for the risk of nausea ( $\mathrm{EOH}: 3.0 \%$ vs. $\mathrm{TOH}: 2.2 \% ; \mathrm{P}=0.545)$ and vomiting $(\mathrm{EOH}: 1.3 \%$ vs. TOH: $0.8 \% ; \mathrm{P}=0.537)$ before drinking after return to the ward. Finally, no significant differences were observed between $\mathrm{EOH}$ and TOH for the rate of nausea $(\mathrm{EOH}: 1.9 \%$ vs. TOH: $1.8 \% ; \mathrm{P}=$ $1.000)$ and vomiting (EOH: $0.4 \%$ vs. $\mathrm{TOH}: 0.2 \% ; \mathrm{P}=0.614)$ after drinking and after return to the ward.

No anastomotic leakages happened in both groups. Six patients $(1.2 \%)$ in the $\mathrm{EOH}$ group experienced postoperative complications (three cases of intestinal fistula, one case of intestinal obstruction, and two cases of postoperative bleeding). However, 11 patients (2.2\%) in the $\mathrm{TOH}$ group experienced postoperative complications (three cases of intestinal fistula, five cases of intestinal obstruction, and three cases of postoperative bleeding). No significant difference was found for the incidence of postoperative complications between the two groups $(P=0.453)$ (Table 5$)$.

\section{Discussion}

Traditional oral intake is allowed until the postoperative ileus resolves and bowel function resumes in patients undergoing gastrointestinal surgery, which is believed to prevent postoperative ileus and protect the surgical anastomoses. However, a previous study showed that the small bowel recovered normal function in 4-8 $\mathrm{h}$ after laparotomy, and gastric emptying resumed on the first postoperative day [14]. Moreover, stomach and pancreas secrete 1-2 $L$ of fluid daily in fasting patients, which showed that patients undergoing colorectal surgery can well tolerate high volumes of fluid [15]. Currently, the efficacy and safety of early oral feeding and traditional oral feeding have already been demonstrated, which suggested that early oral feeding on the first operative day could improve the gastrointestinal recovery parameters, shorten the duration of postoperative ileus, and reduce the length of hospital stay and total postoperative complications in patients undergoing elective colorectal surgery [6,10-12]. Based on the results of above studies, we conducted this prospective randomized controlled trial to determine the effectiveness of EOH versus $\mathrm{TOH}$ for patients who underwent colorectal surgery under general anesthesia. The results of this study indicate that the degrees of thirst scale and oropharyngeal discomfort scale 30 min after drinking were significantly improved, while the time of intestinal movements and safety outcomes between groups were not statistically significant. 
The positive effect of EOH for patients who underwent colorectal surgery was observed in this study. Several studies have reported similar results. Terzioglu [16] and Malhotra et al. [17] show that oral hydration starting within $6 \mathrm{~h}$ after cesarean or gynecologic surgery could help in the quicker return of bowel function. Further, our previous study indicated that $\mathrm{EOH}$ in patients undergoing non-gastrointestinal surgery under general anesthesia was safe, with decreased thirst scale and oropharyngeal discomfort scales and higher satisfaction [13]. However, no data have shown that immediate oral hydration after general anesthesia could be safe in patients undergoing colorectal surgery, although most patients craved for water. Therefore, these effects should be explored to ensure patient comfort after surgery [18]. This prospective trial indicated that $\mathrm{EOH}$ of $0.5 \mathrm{ml} / \mathrm{kg}$ water immediately after recovery from general anesthesia was safe for patients undergoing colorectal surgery, which can effectively relieve thirst and oropharyngeal discomfort without increasing the incidence of nausea, vomiting, and other complications. Compared with previous trials, the time of intestinal movement between groups did not yield significant difference. This could be due to the small volume of water given and the patients did not drink after they went back to the ward until the intestinal function was restored.

A previous study showed that EOH in the PACU was associated with lower incidence of nausea and vomiting following laparoscopic cholecystectomy [19]. However, no significant differences were found between $\mathrm{EOH}$ and $\mathrm{TOH}$ for the incidence of nausea or vomiting and stoma-related complications, including intestinal fistula, intestinal obstruction, and postoperative bleeding. The reason could be the shorter follow-up duration and event rates lower than expected, which did not show a statistical difference and broad confidence intervals, i.e., no statistically significant difference was obtained. Therefore, the current study only provided a relative result for the incidence of safety outcomes between groups.

Several limitations of this study should be mentioned. First, although the balance of baseline characteristics between groups were not statistically significant, several important factors including disease status were not collected, which might affect the effects of EOH and TOH. Second, the stratified analyses for thirst score, oropharyngeal discomfort scale, and the time of intestinal movements due to large variations among involved patients restricted us to conduct a more detailed analysis for the therapeutic effects of oral hydration. Third, the prevalence of adverse events was lower than expected; thus, further large-scale prospective studies are needed to verify the safety profile between EOH and TOH.

\section{Conclusions}

$\mathrm{EOH}$ in patients who underwent colorectal surgery under general anesthesia was considered safe and practical and associated with significantly improved oropharyngeal discomfort and thirst scales. Further, this cohort should be comprehensively assessed and closely monitored for at least $30 \mathrm{~min}$. The long-term effectiveness of optimal timing of oral hydration for patients who underwent colorectal surgery under general anesthesia should be explored.

\section{List Of Abbreviations}


$\mathrm{EOH}$ : early oral hydration; $\mathrm{TOH}$ : traditional oral hydration; PACU: postanesthesia care unit; ASA: American Society of Anesthesiologists.

\section{Declarations}

Ethics approval and consent to participate: This study was approved by the Ethics Committee of Clinical Trials and Biomedical, West China Hospital, Sichuan University (Date: 2012, Number: 220). Written informed consent was obtained from all patients.

Consent to publish: Not applicable.

Availability of data and materials: The datasets analysed during the current study are available from the corresponding author on reasonable request.

Competing interests: The authors declare that they have no competing interests.

Funding: The process of study design, data collection and manuscript writing was funded by Health and Family Planning Commission of Sichuan Province.

Authors' contributions: XY designed the study and analyzed the data. LY analyzed the data and wrote the manuscript. SX and YW collected the data. QL designed the study, analyzed the data and wrote the manuscript. All authors read and approved the final manuscript.

Acknowledgements: Not applicable.

\section{References}

1. van der Pas $\mathrm{MH}$, Haglind $\mathrm{E}$, et al. Laparoscopic versus open surgery for rectal cancer (COLOR II): short-term outcomes of a randomised, phase 3 trial. Lancet Oncol 2013;14:210-8.

2. Moya P, Soriano-Irigaray L, Ramirez JM, et al. Perioperative Standard Oral Nutrition Supplements Versus Immunonutrition in Patients Undergoing Colorectal Resection in an Enhanced Recovery (ERAS) Protocol: A Multicenter Randomized Clinical Trial (SONVI Study). Medicine (Baltimore). 2016;95:e3704.

3. Bisgaard T, Kehlet $\mathrm{H}$. Early oral feeding after elective abdominal surgery - what are the issues? Nutrition 2002;18:944-948.

4. Keele AM, Bray MJ, Emery PW, et al. Two phase randomized controlled clinical trial of postoperative oral dietary supplements in surgical patients. Gut 1997; 40: 393

5. Feng C, Sidhwa F, Cameron DB, et al. Rates and burden of surgical site infections associated with pediatric colorectal surgery: insight from the National Surgery Quality Improvement Program. J Pediatr Surg 2016;51:970-4.

6. Rock JA, Thompson JD. Te Linde's Operative Gynecology. 8th ed. Philadelphia: JB Lippincot-Raven; 1997. p. 142. 
7. Beier-Holgersen R, Boesby S. Influence of postoperative enteral nutrition on postsurgical infections. Gut 1996;39:833

8. Carr CS, Ling KD, Boulos P, et al. Randomised trial of safety and efficacy of immediate postoperative enteral feeding in patients undergoing gastrointestinal resection. BMJ 1996;312:869.

9. Mark S, Schreiner MD. should children Drink before Discharge from Day Surgery. Anesthesiology 1992;76:528-533.

10. Ahmet Dag, TahsinColak, Ozgur Turkmenoglu, et al. A randomized controlled trial evaluating early versus traditional oral feeding after colorectal surgery. Clinics 2011; 66: 2001-2005.

11. Ng WQ, Neill J. Evidence for early oral feeding of patients after elective open colorectal surgery: a literature review. J Clin Nutrs 2006; 15:696-709.

12. Zhuang $\mathrm{CL}$, YeX, Zhang CJ, et al. Early versus Traditional Postoperative Oral Feeding in Patients Undergoing Elective Colorectal Surgery: A Meta-Analysis of Randomized Clinical Trials. Dig Surg 2013;30:225-232.

13. Yin X, Ye L, Zhao L, et al. Early versus delayed postoperative oral hydration after general anesthesia: a prospective randomized trial. Int J Clin Exp Med 2014;7:3491-6

14. Catchpole BN. Smooth muscle and the surgeon.Aust N Z J Surg1989;59:199-208.

15. Moss G. Maintenance of gastrointestinal function after bowel surgery and immediate enteral full nutrition. II. Clinical experience, with objective demonstration of intestinal absorption and motility. J Parenter Enteral Nutr 1981;5:215-220.

16. Terzioglu F, Simsek S, Karaca K, et al. Multimodal interventions (chewing gum, early oral hydration andearly mobilisation) on the intestinal motility following abdominal gynaecologic surgery. $\mathrm{J}$ Clin Nurs 2013; 22: 1917-1925.

17. Malhotra N, Khanna S, Pasrija S, et al. Early oral hydration and its impact on bowel activity after elective caesarean section--our experience. Eur J Obstet Gynecol Reprod Biol 2005;120:53-6.

18. Morag A, Patricia A. Clinical effectiveness in the post-anaesthesia care unit: How nursing knowledge contributes to achieving intended patient outcome. Advanced Nursing 2000; 31:1115-24.

19. Menghang Wu, Lei Yang, Xiaoqi Zeng, et al. Safety and Feasibility of Early Oral Hydration in the Postanesthesia Care Unit After Laparoscopic Cholecystectomy: A Prospective, Randomized, and Controlled Study. J Peroanes Nur 2018; 17 Oct 2018 online.

\section{Tables}

Table 1. Baseline characteristic of the enrolled patients 


\begin{tabular}{|c|c|c|c|}
\hline Variables & $\mathrm{EOH}(\mathrm{n}=473)$ & $\mathrm{TOH}(\mathrm{n}=500)$ & P value \\
\hline Mean age (years) & $58.12 \pm 12.43$ & $59.58 \pm 14.41$ & 0.091 \\
\hline \multicolumn{4}{|l|}{$\operatorname{Sex}(\%)$} \\
\hline Male & $265(56.0 \%)$ & $310(62.0 \%)$ & \multirow[t]{2}{*}{0.059} \\
\hline Female & $208(44.0 \%)$ & $190(38.0 \%)$ & \\
\hline Weight (Kg) & $59.13 \pm 10.31$ & $58.95 \pm 9.82$ & 0.784 \\
\hline \multicolumn{4}{|l|}{ Anesthesia type (\%) } \\
\hline Total intravenous anesthesia & $418(88.4 \%)$ & $421 \square 84.2 \%)$ & \multirow[t]{2}{*}{0.063} \\
\hline Intravenous anesthesia & $55(11.6 \%)$ & $79(15.8 \%)$ & \\
\hline \multicolumn{4}{|l|}{ ASA (\%) } \\
\hline प & $12(2.5 \%)$ & $19(3.8 \%)$ & \multirow[t]{3}{*}{0.178} \\
\hline प & $331(70.0 \%)$ & $324(64.8 \%)$ & \\
\hline प & $130(27.5 \%)$ & $157(31.4 \%)$ & \\
\hline \multicolumn{4}{|l|}{ Surgical sites (\%) } \\
\hline Ascending colon & $56(11.8 \%)$ & $65(13.0 \%)$ & \multirow[t]{6}{*}{0.302} \\
\hline Transverse colon & $18(3.8 \%)$ & $25(5.0 \%)$ & \\
\hline Descending colon & $14(3.0 \%)$ & $11(2.2 \%)$ & \\
\hline Sigmoid colon & $92(19.5 \%)$ & $72(14.4 \%)$ & \\
\hline Rectum & $283(59.8 \%)$ & $313(62.6 \%)$ & \\
\hline Back to the colon & $10(2.1 \%)$ & $14(2.8 \%)$ & \\
\hline Intraoperative blood loss (ml) & $127.42 \pm 94.06$ & $129.21 \pm 108.79$ & 0.784 \\
\hline Intraoperative fluid infusion (ml) & $2073.49 \pm 707.25$ & $2065.46 \pm 713.03$ & 0.860 \\
\hline
\end{tabular}

$\mathrm{EOH}=$ early oral hydration

$\mathrm{TOH}=$ traditional oral hydration

Table 2. Intraoperative drug use between the $\mathrm{EOH}$ and $\mathrm{TOH}$ group 


\begin{tabular}{|c|c|c|c|}
\hline$\overline{\text { ype }}$ & $\mathrm{EOH}(\mathrm{n}=473)$ & $\mathrm{TOH}(\mathrm{n}=500)$ & $P$ value \\
\hline nyl (\%) & $2(0.4 \%)$ & $2(0.4 \%)$ & 1.000 \\
\hline $\operatorname{tanil}(\%)$ & $471(99.6)$ & $495(99.0 \%)$ & 0.453 \\
\hline entanil (\%) & $456(96.4 \%)$ & $485(97.0 \%)$ & 0.72 \\
\hline lurane (\%) & $407(86.0 \%)$ & $418(83.6 \%)$ & 0.326 \\
\hline idol (\%) & $279(59.0 \%)$ & $307(61.4 \%)$ & 0.471 \\
\hline racurium & 391 (82.7\%) & $431(86.2 \%)$ & 0.133 \\
\hline vylcholine chloride (\%) & $6(1.3 \%)$ & $6(1.2 \%)$ & 1.000 \\
\hline गnium (\%) & $42(8.9 \%)$ & $41(8.2 \%)$ & 0.731 \\
\hline onium (\%) & $12(4.8 \%)$ & $24(4.8 \%)$ & 0.064 \\
\hline olam (\%) & $469(99.2 \%)$ & $494(98.8 \%)$ & 0.754 \\
\hline fol (\%) & $471(99.6 \%)$ & $495(99.0 \%)$ & 0.453 \\
\hline igmine (\%) & $293(61.9 \%)$ & $298(59.6 \%)$ & 0.470 \\
\hline Izenil (\%) & $141(29.8 \%)$ & $131(26.2 \%)$ & 0.225 \\
\hline one (\%) & $1(0.2 \%)$ & $1(0.2 \%)$ & 1.000 \\
\hline nethasone (\%) & $34(7.2 \%)$ & $11(2.2 \%)$ & 0.000 \\
\hline Iprednisolone (\%) & $8(1.7 \%)$ & $7(1.4 \%)$ & 0.798 \\
\hline II (\%) & $131(27.7 \%)$ & $113(22.6 \%)$ & 0.076 \\
\hline :etron (\%) & $110(23.3 \%)$ & $119(23.8 \%)$ & 0.880 \\
\hline ietron (\%) & $5(1.1 \%)$ & $4(0.8 \%)$ & 0.747 \\
\hline Isetron (\%) & $312(66.0 \%)$ & $318(63.6 \%)$ & 0.461 \\
\hline
\end{tabular}

$\mathrm{EOH}=$ early oral hydration

$\mathrm{TOH}=$ traditional oral hydration

Table 3. Thirst score, oropharyngeal discomfort scale, and the time of intestinal movements 


\begin{tabular}{lccc}
\hline Outcomes & $\mathrm{EOH}(\mathrm{n}=473)$ & $\mathrm{TOH}(\mathrm{n}=500)$ & $\mathrm{P}$ value \\
& & & \\
\hline Thirst score before drinking & $53.79 \pm 23.39$ & $51.22 \pm 24.18$ & 0.094 \\
\hline Oropharyngeal discomfort scale before drinking & $4.55 \pm 10.22$ & $3.83 \pm 8.18$ & 0.224 \\
\hline Thirst score after 30 minutes of drinking & $45.70 \pm 24.51$ & $62.20 \pm 23.99$ & 0.000 \\
\hline Oropharyngeal discomfort scale after 30 minutes of drinking & $3.71 \pm 8.49$ & $6.18 \pm 11.89$ & 0.000 \\
\hline Intestinal movement & $73.37 \pm 34.49$ & $70.56 \pm 31.71$ & 0.187 \\
\hline
\end{tabular}

$\mathrm{EOH}=$ early oral hydration

$\mathrm{TOH}=$ traditional oral hydration

Table 4. Incidence of nausea and vomiting

\begin{tabular}{lccc}
\hline mes & $\mathrm{EOH}(\mathrm{n}=473)$ & $\mathrm{TOH}(\mathrm{n}=500)$ & $\mathrm{P}$ value \\
?a after 30 minutes drinking (\%) & $9(1.9 \% \square$ & $14(2.8 \% \square$ & 0.403 \\
ing after 30 minutes drinking (\%) & $2(0.4 \%)$ & 0 & 0.234 \\
:a after leaving PACU (\%) & $5(1.1 \%)$ & $10(2.0)$ & 0.301 \\
ing after leaving PACU (\%) & 0 & 0 & \\
?a before drinking after return to ward (\%) & $14(3.0 \%)$ & $11(2.2 \%)$ & 0.545 \\
ing before drinking after return to ward (\%) & $6(1.3 \%)$ & $4(0.8 \%)$ & 0.537 \\
?a after drinking after return to ward (\%) & $9(1.9 \%)$ & $9(1.8 \%)$ & 1.000 \\
ing after drinking after return to ward (\%) & $2(0.4 \%)$ & $1(0.2 \%)$ & 0.614 \\
\hline
\end{tabular}

$\mathrm{EOH}=$ early oral hydration

$\mathrm{TOH}=$ traditional oral hydration

Table 5. Summary of stoma-related postoperative complications 


\begin{tabular}{lccc}
\hline comes & $\mathrm{EOH}(\mathrm{n}=473)$ & $\mathrm{TOH}(\mathrm{n}=500)$ & $\mathrm{P}$ value \\
srall events (\%) & $6(1.3 \%)$ & $11(2.2 \%)$ & 0.453 \\
sstinal fistula (\%) & $3(0.6 \%)$ & $3(0.6 \%)$ & 0.293 \\
sstinal obstruction (\%) & $1(0.2 \%)$ & $5(1.0 \%)$ & \\
stoperative bleeding (\%) & $2(0.4 \%)$ & $3(0.6 \%)$ & \\
\hline
\end{tabular}

$\mathrm{EOH}=$ early oral hydration

$\mathrm{TOH}=$ traditional oral hydration

\section{Figures}

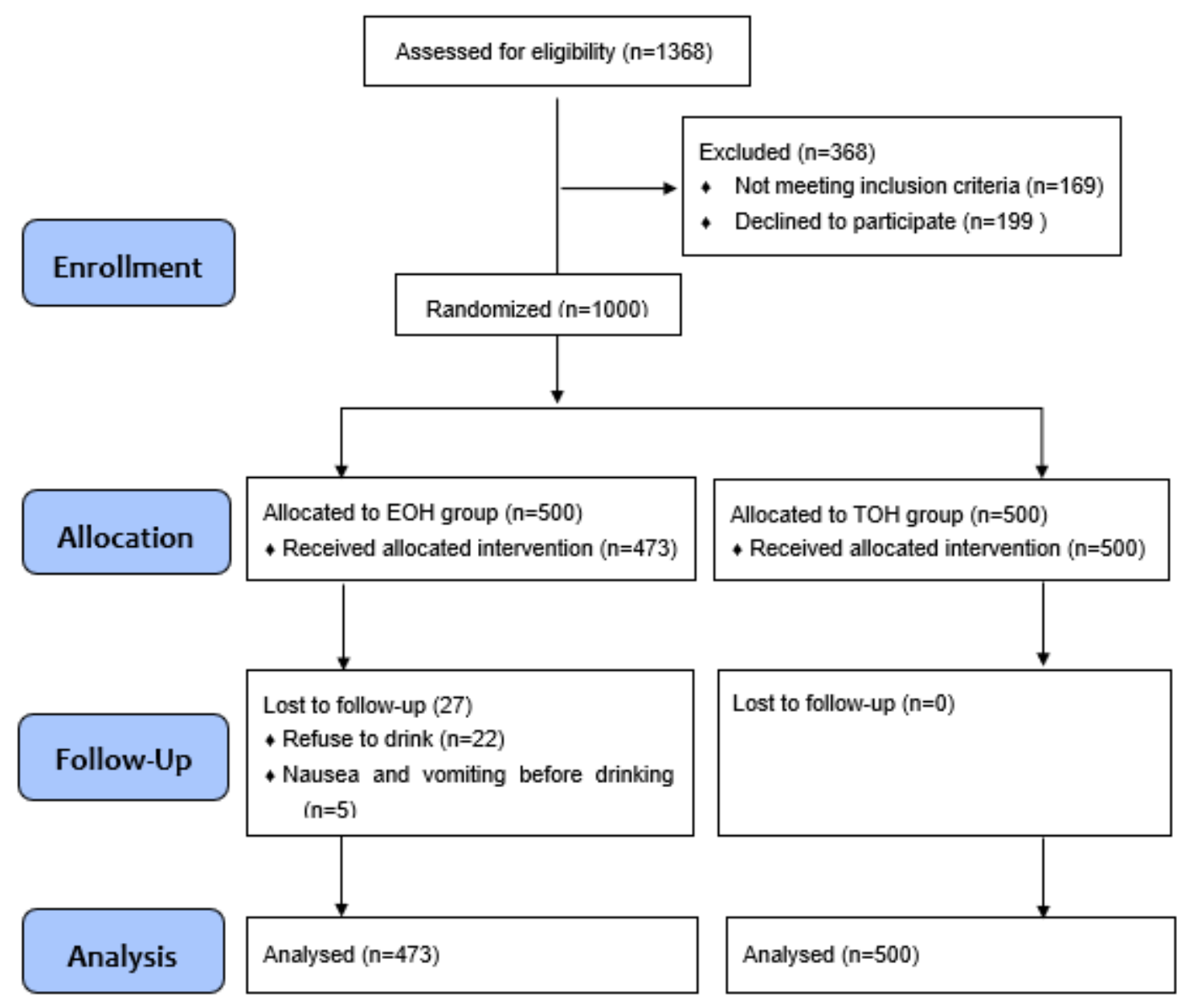

Figure 1 
Study flowchart of patients recruited process. $\mathrm{EOH}=$ early oral hydration $\mathrm{TOH}=$ traditional oral hydration

\section{Supplementary Files}

This is a list of supplementary files associated with this preprint. Click to download.

- CONSORT2010ChecklistMSWord.doc 